solchen zur intravonösen Einspritzung besonders gekennzeichnete Glaser in Anwendung kommen müssen. Ich würde es für zweckmäßig. balten, wenn diese Medikantente ausschlieblich in Flaschen aus blauem Glas (Farbe der Venen!) vom Apotheker geliefert werden müsten.

\title{
Die Notwendigkeit besonderer Gläser für Medikamente zur intravenösen Injektion.
}

Von Dr. Boerner, Facharzt für Chirurgie in Eriurt.

Wahrend für Medikamente, welche per os genommen werden sollen, besondere Gläser vorgeschrieben sind, die in Form und Farbe sich von denjenigen unterscheiden, die für Arzneimiltel zum äußeren Gebrauch bestimmt sind, müssen Medikamente zur intravenösen Injektion in den gleichen Gläsern und mit der gleichen Farbe der Etikette geliefert, werden, wie die als "Aeußerlich" bezeichneten.

Mit der starken Zunahme der Verabreichung von Medikamenten direkt in die Blutbahn ist dies ein unhaltbarer Zustand geworden, und der staat ist verpflichtet, seine Vorschriften der modernen Bẻłandlungsweise anzupassen, um verhängrisvolle Verwechslungen zu ersohweren. Es ist einleuchtend, daß dje versehentliche Einnalme eines Medikamentes zum ätiferen Gebrauch per os oft durch Magenspülungen und andere Maß̉nahmen in seinen Folgen gentildert werden kann; bei einer intravenösen Einspritzung dagegen ist Hilfe meist nicht möglich, weil das Medikament nicht wieder aus dem Körpex entiernt werden kann und die. Wirkung zu schnell eintritt. Daraus folgt, dab mehr noch als bei Arzneien zum inneren Gebrauch bei 